\title{
Improving Error Rate Performance of Correlated Sources by Non-Root-Nyquist Pulse Shaping
}

\author{
Makoto Tanahashi and Hideki Ochiai \\ Department of Electrical and Computer Engineering, Yokohama National University \\ Yokohama, Kanagawa 240-8501, Japan \\ Email: makoto@ochiailab.dnj.ynu.ac.jp; hideki@ynu.ac.jp
}

\begin{abstract}
In band-limited communications, the use of rootNyquist pulse-shaping filters, such as root raised cosine (RRC), is a common premise to guarantee no inter-symbol interference (ISI) in the output from matched filtering. We have recently shown that, for correlated source symbols, some non-root-Nyquist filter is capable of increasing the mutual information of the resulting channel. In this paper, we show that performance improvement can also be achieved in terms of bit error rate.
\end{abstract}

\section{INTRODUCTION}

Information sources, in reality, often exhibit redundancy due to a certain reason such as incomplete source coding. For such a case, there have been a number of approaches proposed to exploiting the redundancy for improving error rate performance. For example, in [1], the authors considered a binary source with unequal probabilities of occurrence and derived the optimal energy allocation for binary symbols. This work was later extended in [2] to sources with memory (i.e., correlated sources). Other abundant examples are found in the field of joint source-channel (JSC) coding, where the source redundancy is taken advantage of for decoding of channel codes (see [3] and references therein).

In this paper, we propose a new approach to exploiting the source redundancy for improving error rate performance. The key idea of the proposed technique is that if the source is correlated and successive symbols have interdependence, the resulting pulse-shaped signal can have a lower average power than that generated from an uncorrelated source. This occurs when the source correlation produces many destructive superpositions of the shaping pulses as illustrated in Fig. 1. Since this reduction in the average transmit power preserves the amplitude of discrete sequences, one can in turn boost the minimum Euclidean distance (i.e., noise margin) of the baseline signal constellation without increasing power consumption at the transmitter. In this regard, the average transmit power reduction obtained in this way can be treated as a gain in SNR. We specifically refer to it as shaping gain $\eta_{s}$ and define it as

$$
\eta_{s}=P_{a v}^{\text {uncor }} / P_{a v}
$$

where $P_{a v}$ denotes the average power observed when filtering a given correlated sequence and $P_{a v}^{\text {uncor }}$ that with respect to uncorrelated sequences. Note that the conventional definition of the shaping gain refers to constellation shaping $[4,5]$, whereas

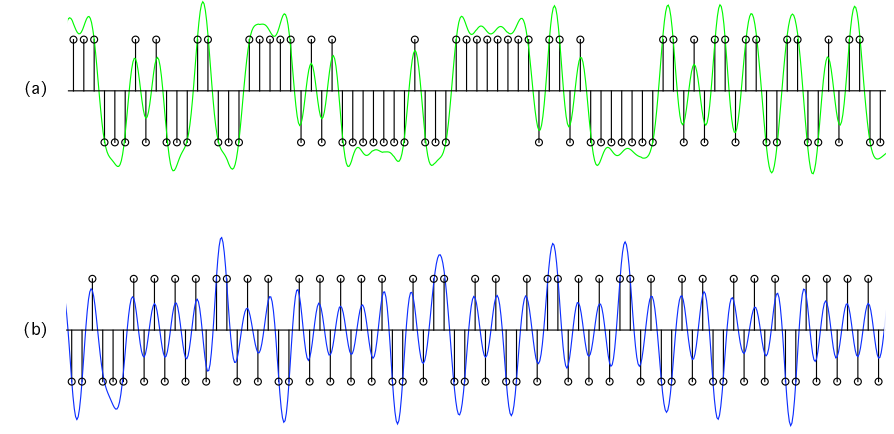

Fig. 1. Examples of transmit signals pulse-shaped by a certain transmit filter. They have different average powers due to different correlation source properties. (a) Uncorrelated symbols result in a unit average power. (b) Correlated symbols can result in a reduced average power.

the shaping gain in our definition is based on the signals after pulse shaping, i.e., the final form of the transmitted signal.

In order to achieve the shaping gain, as we show later, the pulse-shaping filter must not be a root-Nyquist filter: commonly used root raised-cosine (RRC) filters cannot achieve the shaping gain regardless of the correlation property of the source. With this, the channel output is inevitably deformed by inter-symbol interference (ISI) if we presume matched filter reception. Nevertheless, we show that the achievable performance with maximum a posteriori (MAP) demodulation is superior to those with root-Nyquist filters. This suggests that the resulting shaping gain can more than compensate for the loss associated with the ISI.

Recently, the authors have analyzed this system in terms of its mutual information in [6]. The objective of this paper is to further analyze the performance over specific ISI channels and elucidate an achievable gain by evaluating its bit error rate (BER).

The rest of this paper is organized as follows: we describe our system model in the next section. Section III analyzes the shaping gain and Section IV discusses the MAP demodulation for our system model. Simulated error rate performances with several parameter settings are presented in Section V. Finally, Section VI summarizes the findings of this paper. 
(a)

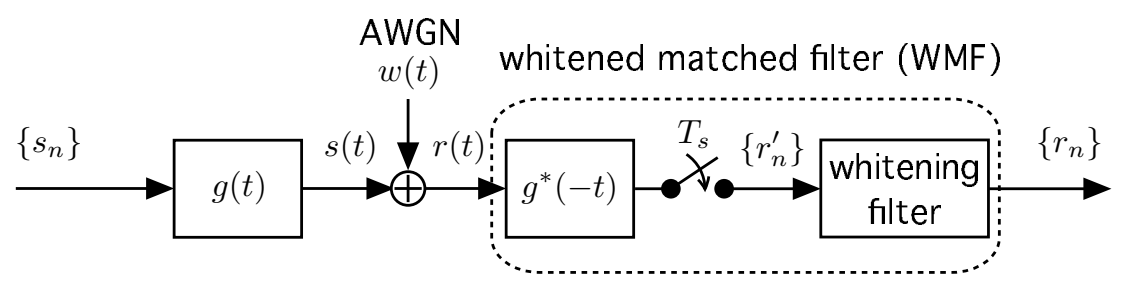

(b)

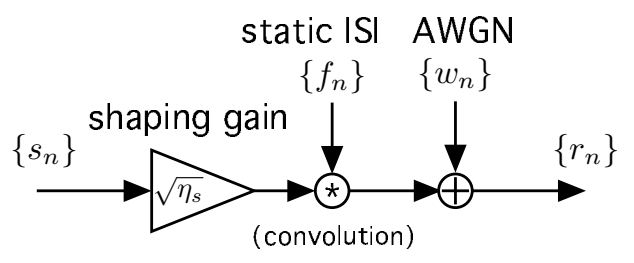

Fig. 2. The channel model considered in this paper. (a) The continuous-time model. (b) Its equivalent discrete-time representation.

\section{System Model}

\section{A. Stochastic Model of Source}

Figure 2 (a) shows the system model considered in this paper. In this figure, $\left\{s_{n}\right\}$ denotes a binary real-valued antipodal (i.e., BPSK) sequence with the average energy (per symbol) of $E_{s}$. We model this sequence as a first-order Markov chain with the state transition matrix defined as

$$
Q=\left[\begin{array}{cc}
1-q & q \\
q & 1-q
\end{array}\right]
$$

Hence, a state moves to the other with the transition probability $q$. We further define that $s_{n}=+\sqrt{E_{s}}$ if the $n$th state is 0 and $s_{n}=-\sqrt{E_{s}}$ for the $n$th state of 1 .

\section{B. Continuous-Time Model}

For band-limitation, $\left\{s_{n}\right\}$ is fed into a pulse-shaping filter $g(t)$ with Nyquist rate $T_{s}$ to form the transmit signal:

$$
s(t)=\sum_{n=-\infty}^{\infty} s_{n} g\left(t-n T_{s}\right) .
$$

We assume that the impulse response $g(t)$ is normalized to have a unit energy, i.e.,

$$
\int_{-\infty}^{\infty}|g(t)|^{2} d t=1
$$

The considered channel impairment between the transmitter and the receiver is only AWGN $w(t)$ with the two-sided noise power spectral density of $N_{0} / 2$. The receiver first performs matched filtering on the received signal $r(t)=s(t)+w(t)$, and its output (after sampled with the interval $T_{s}$ ) is fed into a noise-whitening filter $[7,8]$. Thus, the final output $\left\{r_{n}\right\}$ has maximized signal-to-noise ratio as well as whitened noise which is tractable to develop an optimal demodulator.

\section{Equivalent Discrete-Time Model}

It is convenient for computer simulations to develop an equivalent discrete-time channel model and cast the shaping gain $\eta_{s}$ (to be produced by pulse shaping) as a part of the channel. This discrete-time channel model is shown in Fig. 2 (b). In this model, the input $\left\{s_{n}\right\}$ is first amplified by $\sqrt{\eta_{s}}$ and then convolved with a static ISI $\left\{f_{n}\right\}$ which is the combined impulse response of the matched filter and the whitening filter, i.e., whitened matched filter (WMF) [7,8]. Adding discretetime AWGN samples $\left\{w_{n}\right\}$ to this yields the channel output $\left\{r_{n}\right\}$.

The ISI coefficients $\left\{f_{n}\right\}$ can be calculated from a given $g(t)$ as follows [8]. Let $\phi_{g}[l]$ be the compound impulse response of the matched filters and the subsequent sampler, which is identical to the auto-correlation function (ACF) of $g(t)$ sampled at $l T_{s}$ and thus expressed as

$$
\phi_{g}[l]=\int_{-\infty}^{\infty} g^{*}(t) g\left(t+l T_{s}\right) d t .
$$

We suppose that the effective duration of $\phi_{g}[l]$ spans only over $2 L+1$, i.e.,

$$
\phi_{g}[l]=0, \quad \text { for }|l|>L .
$$

The Z-transform of $\phi_{g}[l]$, which is given by

$$
\Phi_{g}(z)=\sum_{l=-L}^{L} \phi_{g}[l] z^{-l},
$$

has $L$ roots inside the unit circle, namely $\rho_{1}, \ldots, \rho_{L}$. Let $F(z)$ be the polynomial of degree $L$ having these roots:

$$
F(z)=\left(z-\rho_{1}\right) \cdots\left(z-\rho_{L}\right) .
$$

Then the inverse Z-transform of $F(z)$ gives the desired coefficients $\left\{f_{n}\right\}$ of the length $L+1$. With the calculated $\left\{f_{n}\right\}$, 
the input-output relationship can now be formulated as

$$
r_{n}=\sqrt{\eta_{s}} \sum_{l=0}^{L} s_{n-l} f_{l}+w_{n} .
$$

If the pulse-shaping filter is a root-Nyquist filter, then $\phi_{g}[l]=$ $\delta[l]$ (i.e., the Kronecker delta function) and thus $L=0$ and $f_{0}=1$. This setting implies that (9) is reduced to an ISI-free channel.

The receiver retrieves the original data by MAP demodulation based on (9). The specific MAP procedure is given later in detail.

\section{Shaping Gain $\eta_{s}$}

The average power $P_{a v}$ of the signal (3) is given by [6]

$$
P_{a v}=\frac{E_{s}}{T_{s}}\left(1+2 \sum_{l=1}^{L} \phi_{s}[l] \phi_{g}[l]\right)
$$

where $\phi_{s}[l]=\mathrm{E}\left[s_{n+l} s_{n}\right] / E_{s}$ denotes the normalized autocorrelation function (ACF) of $\left\{s_{n}\right\}$. With some algebra, one can find that the ACF $\phi_{s}[l]$ for the settings introduced in Section II-A is given by

$$
\phi_{s}[l]=\operatorname{det}\left(Q^{l}\right), \quad l=1,2,3, \ldots
$$

The other ACF in (10), $\phi_{g}[l]$, is the ACF of $g(t)$ sampled at $l T_{s}$ whose expression is given in (5).

If the source symbols are uncorrelated, i.e., $\phi_{s}[l]=\delta[l]$, the summation in (10) goes to zero and $P_{a v}$ is simply given by $E_{s} / T_{s}$. Thus, the shaping gain defined in (1) is calculated as

$$
\eta_{s}=\left(1+2 \sum_{l=1}^{L} \phi_{s}[l] \phi_{g}[l]\right)^{-1}
$$

This equation suggests that the shaping gain is achievable by using $g(t)$ whose ACF $\phi_{g}[l]$ has the opposite polarity to $\phi_{s}[l]$ to make the summation negative. Likewise, the shaping gain can also become negative in $\mathrm{dB}$ (i.e., loss) when $\phi_{g}[l]$ has the same polarity to $\phi_{s}[l]$ to make the summation positive. To design $g(t)$ that has a desired ACF $\phi_{g}[l]$, the reader is referred to [6]. The above equation also implies that root-Nyquist pulse shapes achieve neither gain nor loss since $\phi_{g}[l]=\delta[l]$ is satisfied for these filters.

\section{MAP DEMODULATOR}

The MAP demodulation of the received sequence $\left\{r_{n}\right\}$ given in (9) can be performed on a trellis diagram that represents Markov process of the source symbols as well as the channel ISI. The state at the $n$th trellis section is defined as

$$
\Gamma_{n} \triangleq\left[s_{n}, s_{n-1}, \ldots, s_{n-L^{\prime}}\right],
$$

where $L^{\prime}=\max (L, 1)$, and thus the number of states is $2^{L^{\prime}}$. The introduction of $L^{\prime}$ is to guarantee that the minimal trellis
TABLE I

SHAPING GAINS (IN DECIBELS) CALCULATED FROM SPECIFIC PARAMETERS OF THE PULSE-SHAPING FILTER AND THE MARKOV SOURCE.

\begin{tabular}{c|c|c|c|c}
\hline & Filter-I & Filter-II & Filter-III & a root-Nyquist filter \\
\hline$q=0.2$ & -0.93 & 1.19 & -1.10 & 0 \\
\hline$q=0.5$ & 0 & 0 & 0 & 0 \\
\hline$q=0.8$ & 1.19 & -0.93 & 2.44 & 0 \\
\hline
\end{tabular}

when $L=0$ (i.e., no ISI case) can still represent Markov process of the source symbols. The Viterbi algorithm is applied to the trellis with a branch metric (BM) which can be derived as

$$
\begin{aligned}
\operatorname{BM}( & \left.\Gamma_{n-1} \rightarrow \Gamma_{n}\right)=\log p\left[r_{n}, \Gamma_{n} \mid \Gamma_{n-1}\right] \\
= & \frac{\left|\sqrt{\eta_{s}} \sum_{l=0}^{L} s_{n-l} f_{l}-r_{n}\right|^{2}}{\left(N_{0} / 2\right)}-\log \operatorname{Pr}\left(s_{n-1} \rightarrow s_{n}\right) \\
& + \text { some constant, }
\end{aligned}
$$

where $p(\cdot)$ denotes the pdf of a given argument. The Markov transition probability $\operatorname{Pr}\left(s_{n-1} \rightarrow s_{n}\right)$ equals to $q$ if $s_{n} \neq s_{n-1}$ and is $1-q$ otherwise. The constant term can be dropped in actual implementation of the MAP demodulator as it does not affect path selection.

\section{ERror Rate Performance}

\section{A. Simulation Parameters}

In conformance with our accompanying work [6], we examine the following non-root-Nyquist filters referred to as Filter-I $(L=1)$, Filter-II $(L=1)$, and Filter-III $(L=2)$ :

Filter-I: $\quad\left\{\phi_{g}[1]\right\}=\{0.2\}$,

Filter-II: $\quad\left\{\phi_{g}[1]\right\}=\{-0.2\}$,

Filter-III: $\left\{\phi_{g}[1], \phi_{g}[2]\right\}=\{0.3,-0.1\}$.

From these parameters and particular transition probabilities $q$ of the Markov source, we can calculate the shaping gain (12) as listed in Table I. Filters-I and III produce shaping gain for $q>0.5$ and Filters-II for $q<0.5$. The shaping gain in turn becomes a loss if $q$ is larger than 0.5 for Filters-I and III (or, smaller than 0.5 for Filters-II). There is neither a gain nor a loss for the Markov source with $q=0.5$ and any value of $q$ for a root-Nyquist filter.

For the examined filters, the discrete-time channel taps $\left\{f_{n}\right\}$ are calculated as

Filter-I: $\quad\left\{f_{0}, f_{1}\right\}=\{0.98,0.20\}$,

Filter-II: $\quad\left\{f_{0}, f_{1}\right\}=\{-0.98,0.20\}$,

Filter-III: $\quad\left\{f_{0}, f_{1}, f_{2}\right\}=\{-0.92,-0.37,0.11\}$.

For a root-Nyquist filter, its single channel tap is given by $f_{0}=1$ with $L=0$ (see Section II-C).

\section{B. Simulation Results}

Figures 3 and 4 contain simulation results of bit error rate (BER) performance as a function of the channel SNR, 


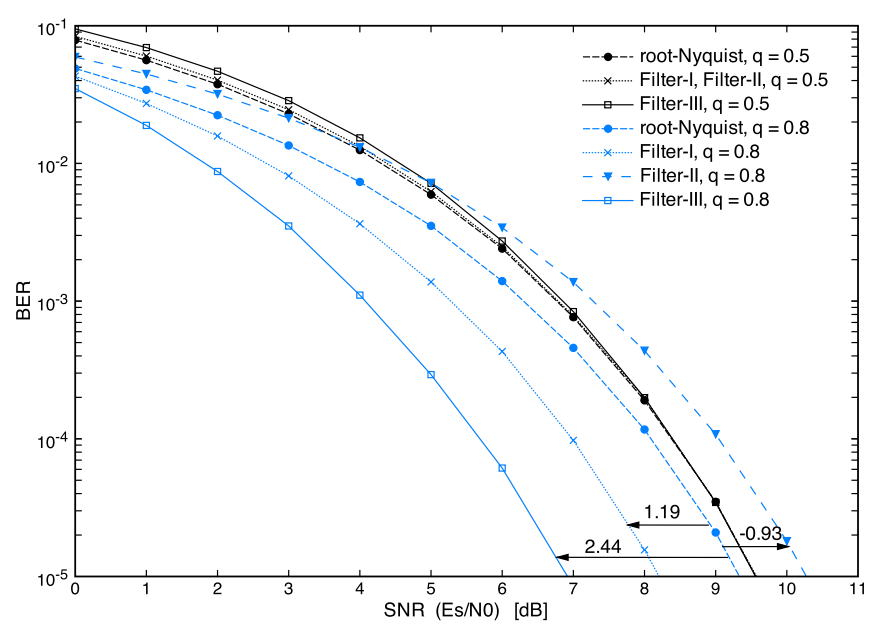

Fig. 3. Bit error rate (BER) performance in the case of $q=0.8$ along with a comparison to the case of $q=0.5$.

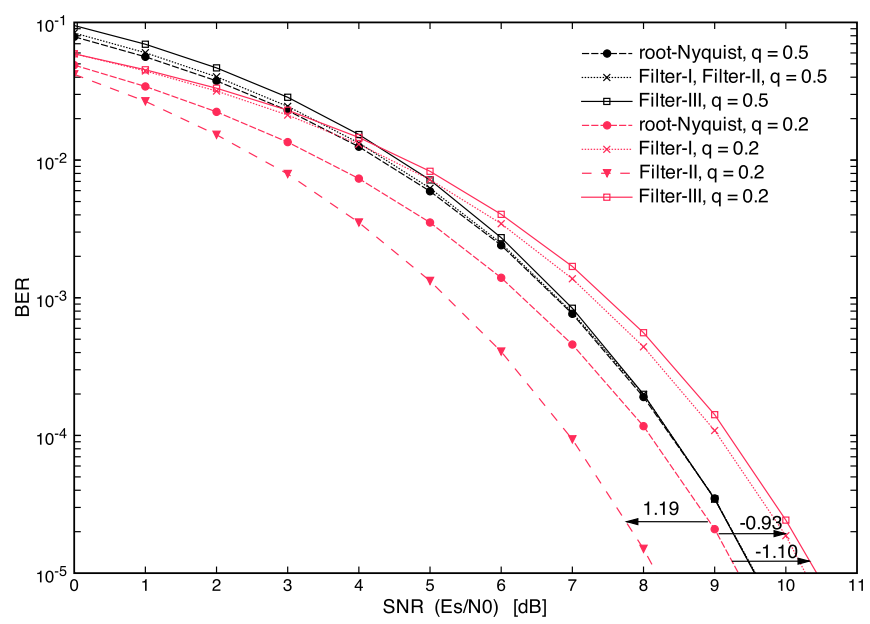

Fig. 4. Bit error rate (BER) performance in the case of $q=0.2$ along with a comparison to the case of $q=0.5$.

$E_{s} / N_{0}$. In the case of $q=0.5$, where the shaping gain is always $0 \mathrm{~dB}$, the use of the non-root-Nyquist filters degrades the performance due to the presence of ISI. (The degradation however diminishes as SNR increases and finally vanishes). Note that, for the combination of a root-Nyquist filter and $q=0.5$, the BER equals to $Q\left(\sqrt{E_{s} / N_{0}}\right)$.
For the correlated cases of $q=0.8$ and 0.2 , even the conventional root-Nyquist system attains some amount of performance gain from MAP demodulation. This is because memory in source symbols contributes to error correction. However, much larger gain is observed for non-root-Nyquist filters that produce shaping gain. Although, in a low SNR region, the performance gain is limited because of the presence of ISI, the asymptotic gain becomes equal to the shaping gain calculated in Table I.

The BER in turn becomes worse than that of the rootNyquist system due to a shaping loss, when the filter's ACF has the same polarities as that of the Markov source. Such cases are observed for Filter-II in Fig. $3(q=0.8)$ and Filter-I and Filter-III in Fig. $4(q=0.2)$.

\section{CONCLUSION}

The use of a non-root-Nyquist filter matched to correlation property of source symbols can save average transmit power and thus achieve shaping gain. With this gain taken into account, we have demonstrated that in some cases achievable BER performance with the MAP demodulator can outperform that of root-Nyquist filter systems.

\section{ACKNOWLEDGEMENT}

This work was in part supported by JSPS Research Fellowships for Young Scientists and MEXT KAKENHI 21760279.

\section{REFERENCES}

[1] I. Korn, J. P. Fonseka, and S. Xing, "Optimal binary communication with nonequal probabilities," IEEE Trans. Commun., vol. 51, pp. 1435-1438, Sept. 2003.

[2] P. M. Crespo, E. Loyo, J. D. Ser, and C. J. Mitchell, "Source controlled modulation scheme for sources with memory," in Proc. IEEE ICC'07, pp. 2619-2624, June 2007.

[3] G. C. Zhu and F. Alajaji, "Joint source-channel turbo coding for binary markov sources," IEEE Trans. Wireless Commun., vol. 5, pp. 1065-1075, May 2006.

[4] G. D. Forney, Jr. and L. F. Wei, "Multidimensional constellations Part I: Introduction, figures of merit, and generalized cross constellations," IEEE J. Select. Areas Commun., vol. 7, pp. 877-892, Aug. 1989.

[5] R. F. H. Fischer, Precoding and Signal Shaping for Digital Transmission. John Wiley \& Sons Inc, 2002.

[6] M. Tanahashi and H. Ochiai, "On destructive superposition of shaping pulses in band-limited linear modulation systems," in Proc. IEEE ISIT'09, July 2009.

[7] G. D. Forney, Jr., "Maximum-likelihood sequence estimation of digital sequences in the presence of intersymbol interference," IEEE Trans. Inform. Theory, vol. 18, pp. 363-378, May 1972.

[8] J. G. Proakis, Digital Communications. McGrawHill, 4th ed., 2001. 